\title{
Cleft Lip and Palate Repair Using a Surgical Microscope
}

\author{
Motoi Kato ${ }^{1}$, Azusa Watanabe ${ }^{1}$, Shoji Watanabe ${ }^{1}$, Hiroki Utsunomiya ${ }^{1}$, Takayuki Yokoyama ${ }^{1}$, \\ Shinya Ogishima ${ }^{2}$ \\ ${ }^{1}$ Department of Plastic and Reconstructive Surgery, Saitama Children's Medical Center, Saitama; ${ }^{2}$ Department of Plastic and Reconstructive \\ Surgery, The University of Tokyo, Tokyo, Japan
}

Background Cleft lip and palate repair requires a deep and small surgical field and is usually performed by surgeons wearing surgical loupes. Surgeons with loupes can obtain a wider surgical view, although headlights are required for the deepest procedures. Surgical microscopes offer comfort and a clear and magnification-adjustable surgical site that can be shared with the whole team, including observers, and easily recorded to further the education of junior surgeons. Magnification adjustments are convenient for precise procedures such as muscle dissection of the soft palate.

Methods We performed a comparative investigation of 18 cleft operations that utilized either surgical loupes or microscopy. Paper-based questionnaires were completed by staff nurses to evaluate what went well and what could be improved in each procedure. The operating time, complication rate, and scores of the questionnaire responses were statistically analyzed.

Results The operating time when microscopy was used was not significantly longer than when surgical loupes were utilized. The surgical field was clearly shared with surgical assistants, nurses, anesthesiologists, and students via microscope-linked monitors. Passing surgical equipment was easier when sharing the surgical view, and preoperative microscope preparation did not interfere with the duties of the staff nurses.

Conclusions Surgical microscopy was demonstrated to be useful during cleft operations.

Keywords Microscope / Cleft lip / Cleft palate

\author{
Correspondence: Motoi Kato \\ Department of Plastic and \\ Reconstructive Surgery, Saitama \\ Children's Medical Center, \\ 1-2 Shintoshin, Chuo-ku, Saitama \\ 330-8777, Japan \\ Tel: +81-48-601-2200 \\ Fax: +81-48-601-2201 \\ E-mail:motoikato25@gmail.com
}

This article contains Supplemental Video S1.

This article was presented at the Japanese Society of Microsurgery on November 16-17, 2016, in Hiroshima, Japan.

\section{INTRODUCTION}

Cleft lip repair and palatoplasty involve a deep, small surgical field that is difficult to observe in detail. Microscopy has been reported to be useful for cleft palate repair [1]; however, no previous reports have focused on its microsurgical merit compared with surgical loupe usage. We investigated the advantages of mi- crosurgery for cleft lip and palate repair and compared microsurgical procedures with conventional surgical procedures to those performed using surgical loupes. In this prospective cohort study, 18 patients with surgically treated cleft lip or palate were classified into 2 equal groups: group $\mathrm{M}$, which underwent microsurgical repair, and group L, which was treated using surgical loupe guidance. The primary outcomes included the oper- 
ative time and complication rate. The experiences of residents and nurses were evaluated via paper-based questionnaires. Prior to using the microsurgical technique for patients, we prepared by using our original training system.

\section{METHODS}

Patients with cleft lip or palate who underwent surgical treatment in Saitama Children's Medical Center between April 2015 and March 2016 were classified into 2 groups: patients who were treated using a surgical microscope (group $\mathrm{M}$ ) and patients who were treated using surgical loupes (group L). Classification was performed randomly by clerks who had no previous knowledge of the severity of the case or the exact diagnosis. Patients with complete cleft lip and palate, syndromic patients (such as those with Robin sequence), and patients with multiple anomalies were excluded. Cleft lip repairs were completed using the Millard method, small triangle method (Onizuka method) [2], and nasoplasty. When indicated, nasal base reconstruction was performed for complete cleft lip cases. All patients with cleft lip were treated at the age of 3 to 4 months. Preoperative nasoalveolar moldings were used for all cleft lip and palate cases. For cleft palates, double opposing Z-plasty was performed in all cases [3]. All cleft palates were classified as Veau type 2. Postoperatively, the same course of care was provided to both groups. Skin sutures were removed from patients with cleft lip 7 days after surgery. Surgical complications such as fistula, wound dehiscence, and infections were carefully assessed after surgery.

All cases were followed up for 1 year postoperatively and the outcomes were reviewed (Table 1). Surgery was performed by a single surgeon for each group. Surgery for group $M$ was performed by a plastic surgeon with 2 years of experience specializing in pediatric plastic surgery. Surgery for group L was performed by a pediatric plastic surgeon with 10 years of experience. Except for the surgeon and the method of magnification, the surgical equipment remained the same in both groups. This study was approved by the Institutional Ethical Board of our hospital and was based on the Strengthening the Reporting of Observational studies in Epidemiology protocol. Informed consent for surgery was obtained.

Prior to surgery for group $M$, the surgeon practiced cutting and suturing techniques under a microscope using an original training system (Figs. 1, 2). The system was simple, with an adult bone model placed upside-down, a paper cup incised on one side, and red-stained gauze attached to simulate the cleft hard palate and mucus.

After completing all the operations analyzed in this study, we distributed a paper-based survey to the operating theater nurses to ascertain the effects of microsurgery on cleft surgery. The questionnaires utilized a scoring system ranging from 1 to 9 ( 1 , worst; 9, best) to evaluate the complexity of preoperative preparation, the difficulty of passing surgical equipment to the sur-

Table 1. Patient list and procedures performed

\begin{tabular}{|c|c|c|c|c|c|c|c|c|c|}
\hline No. & Group & Sex & $\begin{array}{l}\text { Age } \\
\text { (mo) }\end{array}$ & Diagnosis & Classification & Procedure & $\begin{array}{l}\text { Operative time } \\
\text { (min) }\end{array}$ & Complications & $\begin{array}{l}\text { Follow-up } \\
\text { (mo) }\end{array}$ \\
\hline 1 & $\mathrm{~L}$ & Male & 3 & $\mathrm{CL}$ & Incomplete & Cheilo, rhinoplasty & 85 & None & 22 \\
\hline 2 & $\mathrm{~L}$ & Female & 4 & $\mathrm{CL}$ & Incomplete & Cheilo, rhinoplasty & 70 & None & 18 \\
\hline 3 & $L$ & Male & 3 & CLP & Veau 3 & Cheilo, rhinoplasty (nasal base) & 90 & None & 16 \\
\hline 4 & $L$ & Female & 4 & CLP & Veau 3 & Cheilo, rhinoplasty (nasal base) & 123 & None & 14 \\
\hline 5 & $\mathrm{~L}$ & Female & 11 & $\mathrm{CP}$ & Veau 2 & Palatoplasty & 136 & None & 21 \\
\hline 6 & $L$ & Male & 10 & $\mathrm{CP}$ & Veau 2 & Palatoplasty & 115 & None & 16 \\
\hline 7 & $\mathrm{~L}$ & Female & 11 & $\mathrm{CP}$ & Veau 2 & Palatoplasty & 140 & None & 14 \\
\hline 8 & $\mathrm{~L}$ & Female & 12 & $\mathrm{CP}$ & Veau 2 & Palatoplasty & 105 & None & 14 \\
\hline 9 & $L$ & Female & 14 & $\mathrm{CP}$ & Veau 2 & Palatoplasty & 98 & None & 14 \\
\hline 10 & M & Male & 4 & $\mathrm{CL}$ & Incomplete & Cheilo, rhinoplasty & 92 & None & 20 \\
\hline 11 & $M$ & Female & 3 & CLP & Veau 3 & Cheilo, rhinoplasty (nasal base) & 135 & None & 18 \\
\hline 12 & $M$ & Female & 3 & CLP & Veau 3 & Cheilo, rhinoplasty (nasal base) & 162 & None & 17 \\
\hline 13 & $\mathrm{M}$ & Female & 4 & CLP & Veau 3 & Cheilo, rhinoplasty (nasal base) & 75 & None & 14 \\
\hline 14 & $\mathrm{M}$ & Male & 14 & $\mathrm{CP}$ & Veau 2 & Palatoplasty & 87 & None & 14 \\
\hline 15 & $\mathrm{M}$ & Female & 10 & $\mathrm{CP}$ & Veau 2 & Palatoplasty & 127 & None & 14 \\
\hline 16 & $\mathrm{M}$ & Male & 17 & $\mathrm{CP}$ & Veau 2 & Palatoplasty & 130 & None & 14 \\
\hline 17 & $M$ & Male & 12 & $\mathrm{CP}$ & Veau 2 & Palatoplasty & 124 & None & 12 \\
\hline 18 & $\mathrm{M}$ & Male & 12 & $\mathrm{CP}$ & Veau 2 & Palatoplasty & 110 & None & 12 \\
\hline
\end{tabular}




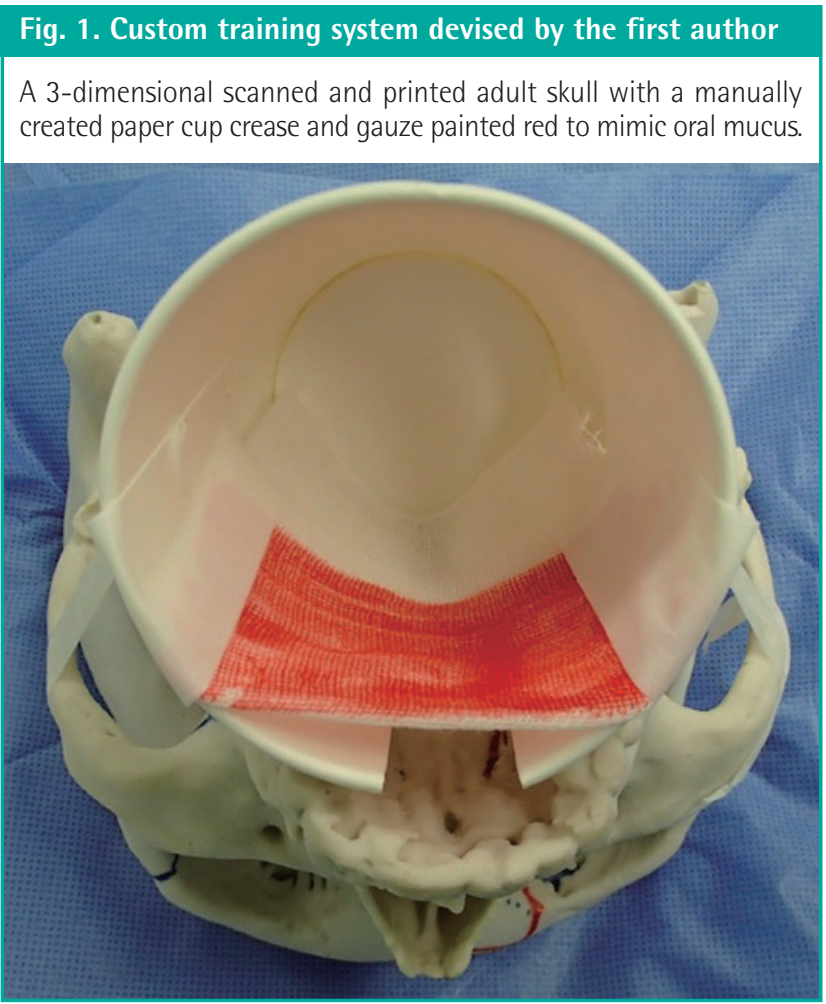

geon as a scrub nurse, and the intraoperative clarity of the surgical site. The paper-based survey was anonymously administered to all 27 staff nurses in the operating theater. Twelve responses obtained from expert nurses were included in this study, including those who had experience with > 10 cases of cleft lip and/or palatoplasty, at least 1 of which was performed under microscopy. Operative time was analyzed using the Mann-Whitney $U$ test. Paper-based survey scores were analyzed using the Wilcoxon signed-rank test.

\section{RESULTS}

Each group included 5 cleft lip patients and 4 cleft palate patients. The age of the patients at the time of surgery ranged from 3 months to 1 year and 5 months (average, 8 months). Eight male (44\%) and 10 female patients were included.

The operative time for group $M$ was 75-162 minutes (mean, 116 minutes), which was not significantly longer than that for group L (70-140 minutes; mean, 107 minutes; $\mathrm{P}=0.56$ ) (Fig. $3)$. The surgical sites were clear, recordable, and reviewable under the microscope (Supplemental Video S1). The surgical procedure improved after several clinical experiences. The surgeon was able to discuss the details with expert surgeons over the monitor, including the regions in the deep and narrow surgical field, which was difficult to observe from outside without a microscope. One year after surgery, none of the patients exhibited
Fig. 2. Custom palatoplasty training system using a surgical microscope

Surgical microscope handling and the cleft palatoplasty procedure can be easily learned via this custom training system.

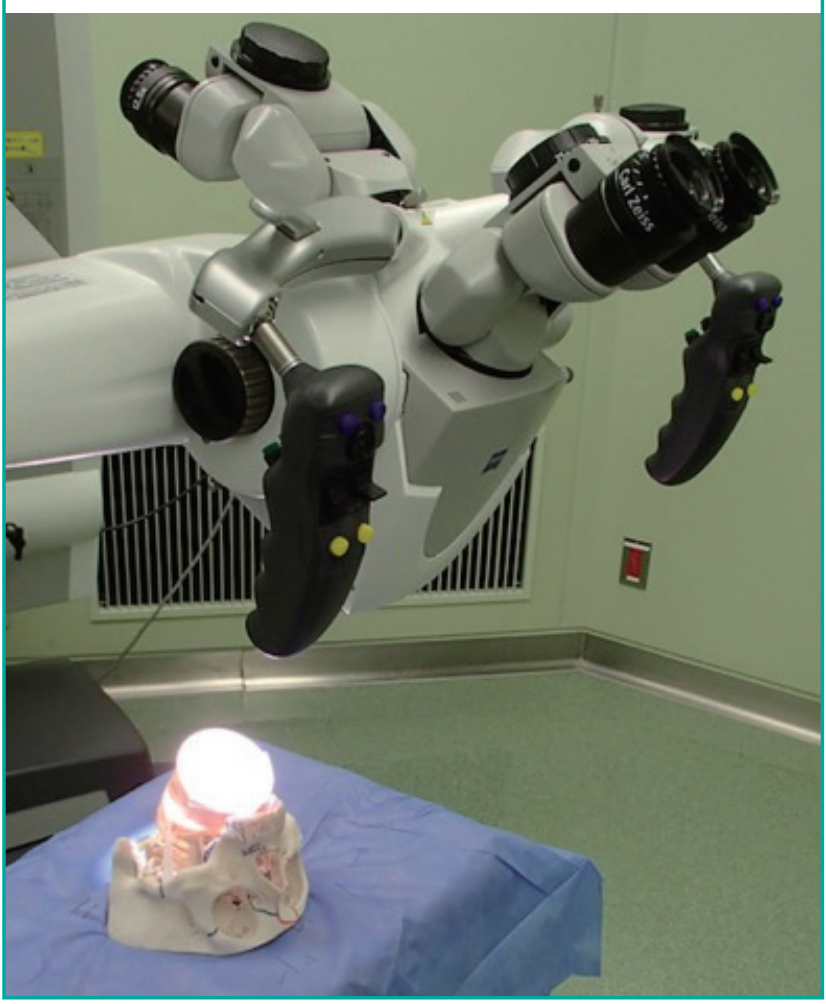

Fig. 3. Operative time comparison between group $L$ and group $\mathrm{M}$

The operative time was not significantly longer for group $\mathrm{M}$ than for group L. Group $M$, underwent microsurgical repair; group L, treated using surgical loupe guidance.

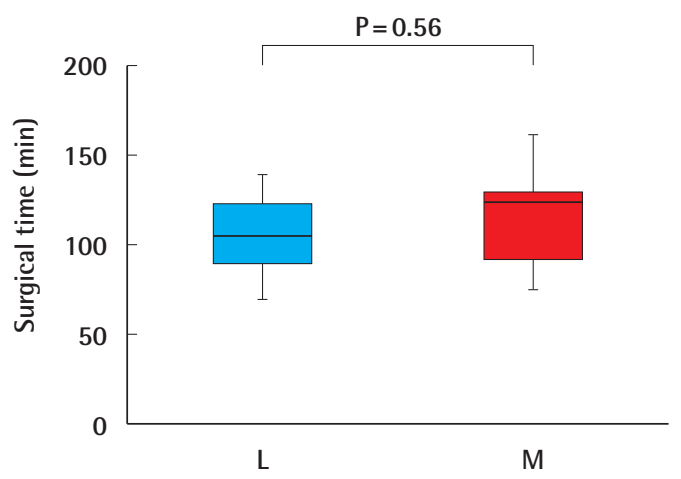

a fistula, wound dehiscence, surgical site infections, or other surgical complications.

The survey of the operating theater nurses demonstrated that the use of microscopy was helpful for them. According to the questionnaire scores, preoperative microscope preparation was 
Fig. 4. Comparison of preparation between group $L$ and group $\mathrm{M}$

Scores assessing the clarity of the surgical field view for nurses, as measured via the paper-based questionnaire (mean, 5.3 vs. 6; $P=0.23$ ). Group $M$, underwent microsurgical repair; group $L$, treated using surgical loupe guidance.

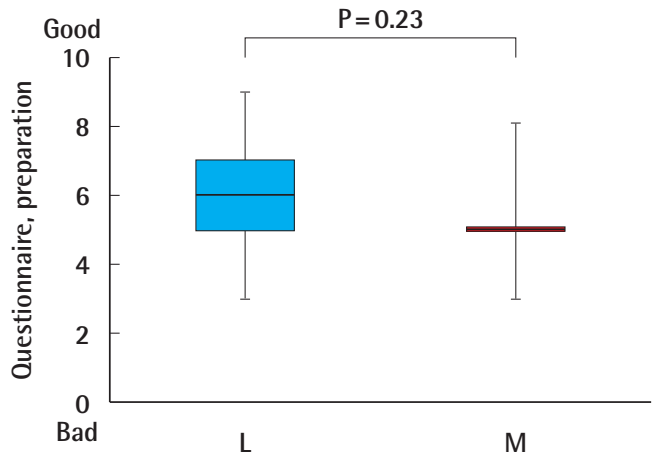

Fig. 5. Equipment handling comparison between group L and group $M$

Scores demonstrating the level of simplicity for scrub nurses to pass surgical equipment during microsurgery, as assessed via the paperbased questionnaire (mean, 7.2 vs. $5 ; \mathrm{P}=0.048$ ). Group $M$, underwent microsurgical repair; group $L$, treated using surgical loupe guidance. ${ }^{*} \mathrm{P}<0.05$.

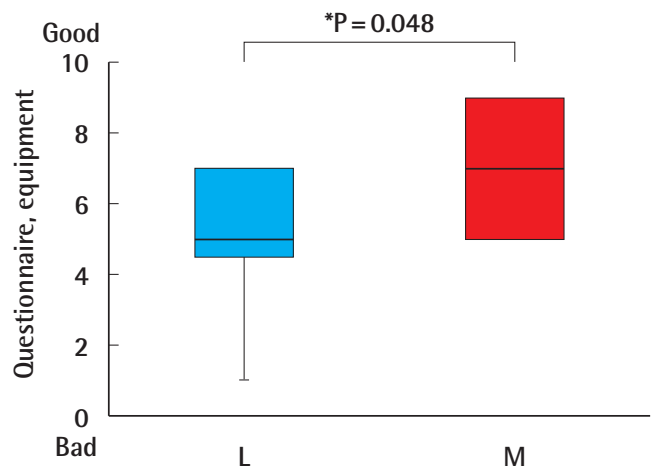

not significantly bothersome (mean, 5.3 vs. 6; $\mathrm{P}=0.23$ ) (Fig. 4), the surgical equipment was more easily passed to the surgeon (mean, 7.2 vs. $5 ; \mathrm{P}=0.048$ ) (Fig. 5), and the surgical site was clearly viewed, which also had the effect of motivating the nurses who were not scrubbed in during their work (mean, 7.8 vs. 2.8; $\mathrm{P}=0.03$ ) (Fig. 6).

\section{DISCUSSION}

In the present study, microsurgery was demonstrated to be useful because it facilitated a clear surgical view without significant-
Fig. 6. Surgical site visualization in group $L$ and group $M$

Scores demonstrating the level of inconvenience for nurses preparing for microsurgery, as assessed via the paper-based questionnaire (mean, 7.8 vs. 2.8; $P=0.03$ ). Group $M$, underwent microsurgical re-

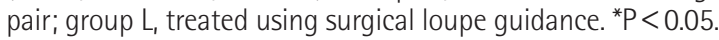

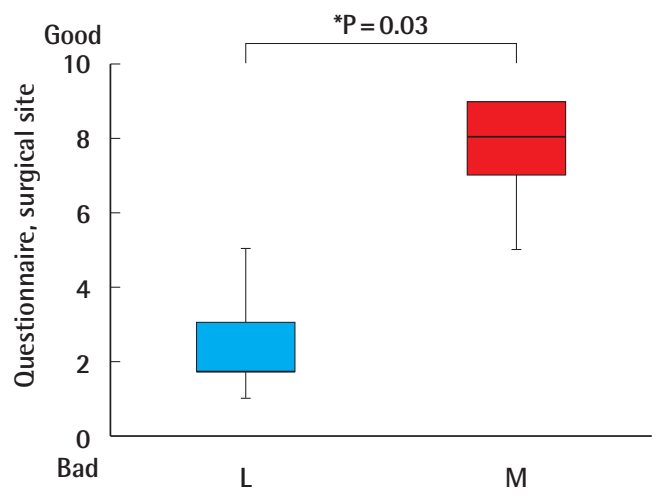

ly increasing the operative time compared to conventional surgical loupe usage. The complication rates were not different between the 2 techniques. Microscopy was also valuable for the education and motivation of nurses by enabling a clear view of the surgical site.

Microsurgery allowed for a clear surgical view. Intraoperatively, the field of view was always bright due to the light from the microscope focusing on the exact area determined by the surgeon. Furthermore, the magnification level was easily adjustable and reliable, and precise procedures, such as separating the levator muscles from the nasal mucosa, were possible. The complication rates did not significantly differ when compared with those of conventional surgical loupes.

Microsurgery is appropriate for delicate procedures [1]. As Sommerlad et al. [4] reported, the margins between the muscle and mucus are easily detectable under a microscope; therefore, precise procedures are possible. Experienced surgeons cause fewer surgical complications than beginning surgeons when using surgical loupes [5-7]; however, in our series of cases, the surgeon who operated on group $\mathrm{M}$, with 1 year of experience, had the same complication rate as the experienced surgeon who operated on group L [8]. Microsurgical procedures may be helpful for reducing the complication rate due to the ability to make precise maneuvers. Furthermore, procedures were easily recorded and reviewed, thereby enabling junior surgeons to improve their skills quickly, which may shorten the learning curve [9].

Microsurgery can improve the education and motivation of operating room nurses by ensuring that they are able to observe the surgical site clearly $[10,11]$. Although we assumed that the preparation of microscopes in the operating room would be 
bothersome for the nurses because they are required to move and set up the microscopes preoperatively, more than $91 \%$ (11 of 12) nurses did not consider it bothersome. Furthermore, sharing the surgical site with staff had vast merits, including making it easy to pass surgical equipment, facilitating a deeper understanding of the procedure, and enabling better cooperation.

Microsurgery is particularly useful for deep and short surgical fields such as cleft palate, gingiva, and nasal base reconstruction for cleft lips. Therefore, microsurgery may be advantageous for pharyngeal flap surgery. Because 3-dimensional structures are somewhat difficult to observe under the microscopic view, careful reconstruction during nasoplasty is required. For the surgical operators, the microscope helped reduce the strain on the neck during the surgical procedure for the anterior part of the palate. When using surgical loupes, surgeons have to bend forward and look down to manipulate the anterior parts. Although there are deflection models of surgical loupes available that can reduce the stress on the surgeon's neck, the surgeon still needs to bend for a better view. However, when using a microscope, surgeons can keep looking straight when manipulating either the anterior or posterior part of the cleft because the angle of the microscope can be adjusted.

This study involved a small number of cases, which limits the generalizability of our results. Furthermore, only short-term results were assessed. According to previous reports describing the advantages of microscopy for cleft palate surgery, the longterm outcomes of microsurgical procedures were superior to those of conventional procedures [1,4]. In our present study, the follow-up time was not long enough to assess objective velopharyngeal function after surgery. Additionally, the questionnaire was answered by the same group of nurses for each group, resulting in correlations. Therefore, the statistical analysis was performed with related data.

Surgeons have used microscopy during reoperations for cases with unsatisfactory outcomes after a conventional procedure [4]. To complete microsurgical cleft lip repair and palatoplasty, surgeons must be familiar with handling a microscope. Our original training system was helpful for preoperatively teaching the surgeon how to handle the microscope. Simulator models are available [12], and even robotic surgery is considered to be practical [13].

Using a microscope during cleft lip surgery and palatoplasty was useful because the surgical field was clearly observable, precise procedures were possible, residents and nurses felt that the procedure was educational, and the operative time did not significantly increase.

\section{CONFLICT OF INTEREST}

No potential conflict of interest relevant to this article was reported.

\section{PATIENT CONSENT}

The patient provided written informed consent for the publication and the use of their images.

\section{REFERENCES}

1. Sommerlad BC. The use of the operating microscope for cleft palate repair and pharyngoplasty. Plast Reconstr Surg 2003;112:1540-1.

2. Onizuka T. A new method for the primary repair of unilateral cleft lip. Ann Plast Surg 1980;4:516-24.

3. Furlow LT Jr. Cleft palate repair by double opposing Z-plasty. Plast Reconstr Surg 1986;78:724-38.

4. Sommerlad BC. A technique for cleft palate repair. Plast Reconstr Surg 2003;112:1542-8.

5. Rossell-Perry P. Flap necrosis after palatoplasty in patients with cleft palate. BioMed Res Int 2015;2015:516375.

6. Daniels KM, Yu EY, Maine RG, et al. Palatal fistula risk after primary palatoplasty: a retrospective comparison of humanitarian operations and tertiary hospitals. Lancet 2015;385 Suppl 2:S37.

7. Schonmeyr B, Wendby L, Campbell A. Early surgical complications after primary cleft lip repair: a report of 3108 consecutive cases. Cleft Palate Craniofac J 2015;52:706-10.

8. Paine KM, Tahiri Y, Wes AM, et al. An assessment of 30-day complications in primary cleft lip repair: a review of the 2012 ACS NSQIP Pediatric. Cleft Palate Craniofac J 2016;53:283-9.

9. Smarius B, Breugem C. Surgical learning curve in performing palatoplasty: a retrospective study in 200 patients. J Craniomaxillofac Surg 2015;43:1868-74.

10. Allori AC, Mulliken JB, Meara JG, et al. Classification of cleft lip/palate: then and now. Cleft Palate Craniofac J 2017; 54:175-88.

11. Sandham A. Classification of clefting deformity. Early Hum Dev 1985;12:81-5.

12. Podolsky DJ, Fisher DM, Wong KW, et al. Evaluation and implementation of a high-fidelity cleft palate simulator. Plast Reconstr Surg 2017;139:85e-96e.

13. Podolsky DJ, Fisher DM, Wong Riff KW, et al. Infant robotic cleft palate surgery: a feasibility assessment using a realistic cleft palate simulator. Plast Reconstr Surg 2017;139:455e$465 \mathrm{e}$. 
Supplemental Video S1. The surgical sites were clear, recordable, and reviewable under the microscope.

Supplemental data can be found at: http://e-aps.org/src/sm/aps-44-490-s001.mp4 